\title{
A New Fuzzy Rule Based Contrast Enhancement Method using The Two-Steps Automatic Clustering Algorithm
}

\author{
Ha CHE-NGOC ${ }^{1, *}$, Anh-Thy PHAM-CHAU ${ }^{2,3}$, Dibya Jyoti BORA ${ }^{4}$
}

${ }^{1}$ Faculty of Mathematics and Statistics, Ton Duc Thang University, Ho Chi Minh City, Vietnam

${ }^{2}$ Division of Computational Mathemactics and Engineering, Institite for Computational Science, Ton Duc Thang University, Ho Chi Minh City, Vietnam

${ }^{3}$ Faculty of Mathematics and Statistics, Ton Duc Thang University, Ho Chi Minh City, Vietnam

${ }^{4}$ Department of Computer Science \& Applications, Barkatullah University, Bhopal, India

*Corresponding Author: Ha CHE-NGOC (email: chengocha@tdtu.edu.vn)

(Received: 16-November-2018; accepted: 30-December-2018; published: 31-December-2018)

DOI: http://dx.doi.org/10.25073/jaec.201824.214

\begin{abstract}
The contrast is a major factor influencing the image quality; therefore, image contrast enhancement technique is more and more widely applied in the field of image processing. In this paper, a new fuzzy rule-based contrast enhancement method using the two-steps automatic clustering algorithm is proposed. Specifically, based on the Automatic clustering algorithm, a state-of-art method in cluster analysis and data mining, this paper proposes a two-steps Automatic clustering method to determine the number of fuzzy sets and locate the critical point in membership functions so that they are suitable for the distribution of pixel intensity values. The experiments on the "Lena" image and other natural images demonstrate that the new method can effectively enhance the contrast of the images and meet the demands of human eyes perception at the same time.
\end{abstract}

\section{Keywords}

contrast enhancement, automatic clustering, fuzzy rule, natural image.

\section{Introduction}

The contrast of an image is the difference in luminance or intensity and is a very important characteristic which decides the quality of image [1]. The contrast enhancement (CE) technology is an indispensable and essential technique to improve the image quality and visual effect so that the processed image can be better than the original image. It is applied in medical image processing, remote sensing and digital image processing and so forth.

In literature, there exist several $\mathrm{CE}$ techniques that have been proposed for image contrast enhancement. The simplest method is the global stretching or normalization technique. Given an original image, with the intensity values in the interval $[a, b]$, this method enhances the image by normalizing the intensity values to the new interval $\left[a^{\prime}, b^{\prime}\right],\left(a^{\prime} \leq a<b \leq b^{\prime}\right)$. However, because this method only utilizes a linear scaling function to the image, the enhancement is less harsh. Another technique which is commonly used in contrast enhancement is histogram equalization [2]. This method transforms a low contrast image to high contrast image by distributing the components of the histogram to cover a wide range of gray scale, which 
approximates uniform distribution. Some related histogram based enhancement methods, which were introduced, such as bi-histogram equalization, multi-histogram equalization, contrast limited adaptive histogram equalization, histogram specification, etc. [3-8]. Nevertheless, histogram equalization and its related works have a drawback when the mean intensity value of the image is shifted to the middle gray-level of the intensity range and may be unsuitable for the human eyes perception. Thus, histogram equalization based techniques are not useful in the case where brightness preservation is required [9].

In 1965, Zadeh first established the foundation of fuzzy set theory where everything is a matter of degree [10]. Fuzzy logic not only recognizes true and false values but also is useful for propositions that can be represented with varying degrees of truth and falseness. Fuzzy logic has filled an important gap in engineering design methods left vacant by purely mathematical approaches. As a result, fuzzy logic has become a well-established topic in both theoretical and practical aspects [11-16]. In image processing, fuzzy rule-based contrast enhancement is one of the most efficient methods for gray/color image contrast enhancement. Generally, a CE technique using fuzzy logic approach consists of three steps: fuzzification, modification and defuzzification [9]. In fuzzification step, the membership functions are established to transform the image intensity values to the membership degrees whose value ranges between 0 and 1 . In the modification step, the membership values are then combined with the defined fuzzy set operations to get the weight of each fuzzy rule. In defuzzification step, based on the defined methods, the qualified output results are combined so that the crisp output is received. According to these steps, numerous effective fuzzy logic algorithms were proposed for image contrast enhancement with different number of fuzzy sets and different kinds of fuzzy membership functions. Hassanien et al. [17] utilized three fuzzy sets: dark, gray and bright combined the triangular membership functions to transform the intensities into fuzzy sets; Kannan et al. [1] utilized three fuzzy sets combined the Gaussian membership function; Kansal and Nitin Kumar [18], utilized two fuzzy sets: dark and bright combined the ramp Gaussian membership function; Preethi [19] utilized two fuzzy sets combined the square or cube membership functions; Yun et al. [20] utilized one fuzzy set with two different kinds of membership function based on the threshold $m$. Obviously, the key to the fuzzy rule-based contrast enhancement is setting up suitable parameters such as the number of fuzzy sets, the value of critical points, the shape of membership functions, etc. Although above methods have contributed to enhancing image, the number of fuzzy sets, the choice of membership functions and the value of critical points have been fixed according to the experiences. Hence, these methods are not suitable when dealing with various kinds of images. For instance, given an image has two areas including bright and dark domains, the method utilizing three fuzzy sets may present a low performance. This problem is mentioned and overcome by [21]. However, the fuzzy clustering method, FCM, of [21] just contributed to determining the critical points but did not identify how many parts (fuzzy sets) the image contains.

In order to fill the researched gaps mentioned above, in this paper, we stress on the importance and necessity of having an automatic method for contrast enhancement and propose a new fuzzy rule-based contrast enhancement method using the two-steps automatic clustering algorithm. The automatic clustering algorithm (AC) [22], which has been an interesting issue in recent years [22-28], has effectively solved the problems about determining the number of clusters and the cluster centroids. Based on this characteristic, the AC can be directly performed for determining the number of fuzzy sets and locating the critical points in $\mathrm{CE}$ technique. Nevertheless, to the best of our knowledge, none of the previous study has investigated a $\mathrm{CE}$ technique using the AC. In case of one-dimension, the AC is an algorithm with complexity $O\left(n^{2}\right)$; therefore, it is vulnerable to a huge number of variables, namely, in case of $\mathrm{CE}$, the number variables or the number of pixels is too huge to apply the $\mathrm{AC}$. We, at first, also try to apply the original $\mathrm{AC}$ to $\mathrm{CE}$ but it leads to a large amount of calculation. Consequently, the basic objective of this paper is not only to automatically determine 
the number of fuzzy sets and locate the critical points but also to significantly reduce the computational cost and has a good real-time performance. Therefore, we propose a new method, called the two-steps Automatic clustering algorithm (2-AC), for determining the number of fuzzy sets and locating the critical points that would next be utilized in $\mathrm{CE}$ techniques. The new method mainly includes two steps. In the first step, we divide the whole image into sliding windows and apply the $\mathrm{AC}$ to each sliding window to get the local centroids. In the second step, the AC continues to be applied to the set of all local centroids to obtain the final centroids or the critical points position. Based on the number of obtained centroids, the number of fuzzy sets is also determined. In the best case, the $2-\mathrm{AC}$ can significantly reduce the algorithm complexity to $O\left(n^{4 / 3}\right)$. The whole new $\mathrm{CE}$ technique, including the phases of fuzzification, modification and defuzzification, is now denoted as $2-\mathrm{ACCE}$, is illustrated and tested by the wellknown Lena and other natural images based on the visualization, the root mean square (RMS) and the Measure of Enhancement (EME) criteria. The obtained results show that the 2-ACCE not only has the best RMS, EME values among the other three conventional methods but also well maintains the image information and makes the enhanced image details more clear and vivid.

The rest of this paper is organized as follows. A review of image contrast enhancement using fuzzy logic and the criteria to evaluate the quality of the processed images is presented in Section 2. The Automatic clustering and the proposed method are presented in Section 3. The experimental results are shown in Section 4, and Section 5 is the conclusion.

\section{Preliminary}

\subsection{Contrast enhancement using fuzzy logic}

In brief, the image contrast enhancement using fuzzy logic approach has three main steps: fuzzification, modification and defuzzification.

\section{Fuzzification}

Given $c$ fuzzy sets with corresponding membership functions and critical points. Through the membership functions, the input intensity values are transformed to membership degrees by one-to-one mapping. Some typical fuzzification methods with different number of fuzzy sets and membership functions, which were designed for image contrast enhancement, are provided by Figure 1. For example, based on Figure 1a, a pixel whose intensity value from 0 to 0.25 is completely assigned to the dark set, a pixel whose intensity value from 0.75 to 1 is completely assigned to the bright set, a pixel point whose intensity value $g=0.4$ is assigned to the dark, gray, bright sets with membership values by 0.4 , 0.6, 0, respectively. Obviously, when utilizing different number of fuzzy sets, membership functions and critical points, as Figure 1b, 1c, 1d, the above intensities will obtain different membership degrees. Therefore, the choice of number fuzzy sets, type of membership functions and critical points are really important factors influencing the output contrast enhancement and need to be paid attention to. (a)

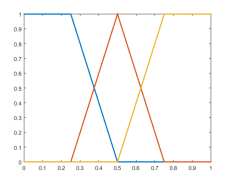

(c)

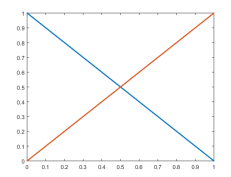

(b)

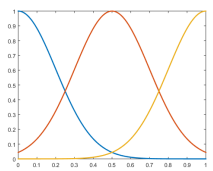

(d)

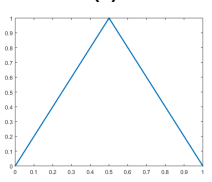

Fig. 1: Some of typical fuzzification methods

\section{Modification}

Without loss of generality, we suppose to have 3 fuzzy sets: dark, gray and bright. The modification step is performed by the following rules:

- If dark then black. 
- If gray then gray.

- If bright then white.

The purpose of modification step is making a dark pixel become darker, a gray pixel become mid-gray and a bright pixel become brighter. For this purpose, [1] presented a simple and effective method to modify the critical points: $V_{1} \rightarrow V_{1}^{\prime}=0, V_{2} \rightarrow V_{2}^{\prime}=0.5, V_{3} \rightarrow V_{3}^{\prime}=1$, where $V_{1}, V_{2}, V_{3}$ are the critical points of the three fuzzy sets. In general, let $c$ be the number of fuzzy sets in an image, the following rule is utilized for modification step:

$$
\left\{\begin{array}{l}
V_{1} \rightarrow V_{1}^{\prime}=0 \\
V_{i} \rightarrow V_{i}^{\prime}=i /(c-1)
\end{array}\right.
$$

where $i=1, \ldots, c$ and the intensity value is normalized to interval $[0,1]$.

\section{Defuzzification}

The aim of this step is to generate new intensity values. Let $g$ is the original intensity value in the image, the output intensity value $g^{\prime}$ can be calculated as follows.

$$
g^{\prime}=\frac{\sum_{i=1}^{c} \mu_{i}(g) V_{i}^{\prime}}{\sum_{i=1}^{c} \mu_{i}(g)}
$$

where $\mu_{i}(g)$ denotes the membership function value for assigning $g$ to fuzzy set $i$ in the fuzzification step and $V_{i}^{\prime}$ denotes the modified critical point of fuzzy set $i$ in the modification step.

\subsection{Contrast enhancement evaluation metrics}

Some criteria often used for measuring the enhanced image quality are presented as follows.

\section{Weber contrast}

The Weber contrast $\left(W_{c}\right)$ is defined as:

$$
W_{c}=\frac{I-I_{b}}{I_{b}}
$$

where $I$ and $I_{b}$ are respectively the object and the background intensities.

\section{Michelson contrast}

The Michelson contrast $\left(M_{c}\right)$ is defined as:

$$
M_{c}=\frac{I_{\max }-I_{\min }}{I_{\max }+I_{\min }}
$$

where $I_{\max }$ and $I_{\min }$ are respectively the maximum and minimum intensity values in the image.

\section{Root mean square}

The Root mean square (RMS) contrast is defined as:

$$
\mathrm{RMS}=\sqrt{\frac{\sum_{i=1}^{r} \sum_{j=1}^{c} I_{i j}-\mu(I)}{r c}},
$$

where $r, c$ are the number of rows and columns of the image, $I_{i j}$ is the intensity of pixel at position $(i, j), \mu(I)$ is the mean of all the intensity values in the entire image.

\section{Measure of Enhancement}

The Measure of Enhancement (EME) contrast is defined as:

$$
\mathrm{EME}=\frac{1}{B_{1} B_{2}} \sum_{i=1}^{B_{1}} \sum_{j=1}^{B_{2}} 20 \ln \left(\frac{I_{i j}^{\max }}{I_{i j}^{\min }+c}\right)
$$

where $B_{1} B_{2}$ is the number of blocks the image is divided into, $I_{i j}^{\max }$ and $I_{i j}^{\max }$ are respectively the maximum and minimum intensity values in block $(i, j), c$ is a constant that help to avoid the denominator of zero.

Among the above criteria, the Weber contrast can be applied on the image with a large, uniform background; the Michelson contrast can be applied on the image where both bright and dark features are equivalent [19]. Hence, both of them are not suitable for the natural images [29] but are the source of inspiration for numerous other criteria. The RMS contrast computes the standard deviation of image intensity; hence, it can be considered as a measure of global contrast. The EME, which gets inspired from the Weber and the Michelson contrast, computes the intensity variation for each block to see the contrast of 
the local area and then computes the average of them. Therefore, the EME can be considered as a measure of global contrast. As numerous studies before [19, 29-31], in this paper, the quality of the enhanced image is evaluated by the RMS and the EME, where the higher RMS and EME indicate the higher image contrast.

\section{The automatic clustering algorithm and the proposed method}

As mentioned above, in literature, the choice of membership function shapes, the number of fuzzy sets, and the location of critical points are fixed by author experiences but are not fixed by the number of different segments of grayscale. As a result, it makes these methods may be not suitable when dealing with various types of image. To overcome this drawback, this paper puts forward a new fuzzy logic based contrast enhancement method using two-steps Automatic clustering algorithm. The Automatic clustering algorithm (AC), the two-steps Automatic clustering algorithm (2-AC), which is a modification of the AC designed to deal with the problem of finding the number of fuzzy sets and the critical points in a large size image, and the new contrast enhancement method using two-steps Automatic clustering algorithm (2-ACCE) are presented in the following subsections.

\subsection{The automatic clustering}

The Automatic clustering proposed by [22] is one of the state-of-art data mining methods and is being efficiently applied in different areas. This method can make discrete elements automatically converge to the centroids of the true clusters so that we can determine the correct number of clusters and cluster centroids in a reasonable way. That is to say, this method can apply to identify the actual number intensity groups as well as the group centroids in an image. Based on the obtained result, we can determine the number of fuzzy sets as well as the critical point locations. The original Automatic clustering algorithm, AC, was proposed to solve the problem of the fuzzy number while the intensity value is the real number. Therefore, this paper first provides the special version of $\mathrm{AC}$ that only solve the problem of real number to save the computational cost. Given data set $X=\left\{x_{1}, x_{2}, \ldots, x_{n}\right\}$, the AC algorithm, the Algorithm 1, divides $X$ into $k$ clusters, where the number of clusters $k$ and the cluster centroids are determined, automatically.

The $\mathrm{AC}$ is a flexible algorithm due to the fact that we not only can determine the number of clusters automatically but also can control the number of clusters depending on the value of $\lambda$; hence, it is suitable for different kinds of processing and various applications. For instance, each of the data points is its own cluster as $\lambda \rightarrow 0$ and the data have only one cluster as $\lambda \rightarrow+\infty$; when having no prior information about the number of clusters, we often choose $\lambda=d s / 10$, then the number of clusters will be determined, automatically $[22,25]$. In the $\mathrm{AC}$ algorithm, the function $K$ decides the influence between $v_{i}$ and $v_{j}$. For example, if $v_{i}=v_{j}$ then $d\left(v_{i}, v_{j}\right)=0$ and $K=\exp (0)=1$; if the distance between $v_{i}$ and $v_{j}$ is larger than $d_{s}, K$ is equal to 0 , that is, $v_{i}$ is not affected by its extreme elements. In the $\mathrm{AC}$, elements move automatically according to the impact of all the other elements, especially of some nearest neighbors. Therefore, the elements in the same cluster can be converged into the same value through the iterative calculation by Formula 2. At last, the final number of clusters and the cluster centroids can be determined automatically. Thus, the AC algorithm has more advantages compared to conventional clustering techniques like $k$-means and FCM, in which the number of clusters is user-defined. The automatic characteristic of the $\mathrm{AC}$ algorithm can be directly performed for determining the number of fuzzy sets and locating the critical points in $\mathrm{CE}$ techniques. Unfortunately, the $\mathrm{AC}$ algorithm needs to calculate the pairwise distance between all elements of the data set for processing; hence, in case of one-dimension, the $\mathrm{AC}$ is an algorithm with complexity $O\left(n^{2}\right)$; as a result, it is vulnerable to huge number of variables, or the number of pixels, and is impossible to apply to CE. We, at first, also try to apply the origi- 
nal $\mathrm{AC}$ to $\mathrm{CE}$ but it leads to an extremely high computational cost. Obviously, the key is the reducing the computational cost in the procedure. Therefore, we propose another AC-based method for determining the number of fuzzy sets and locating the critical points that would next be utilized in $\mathrm{CE}$ techniques. The new method is presented in the following subsection.

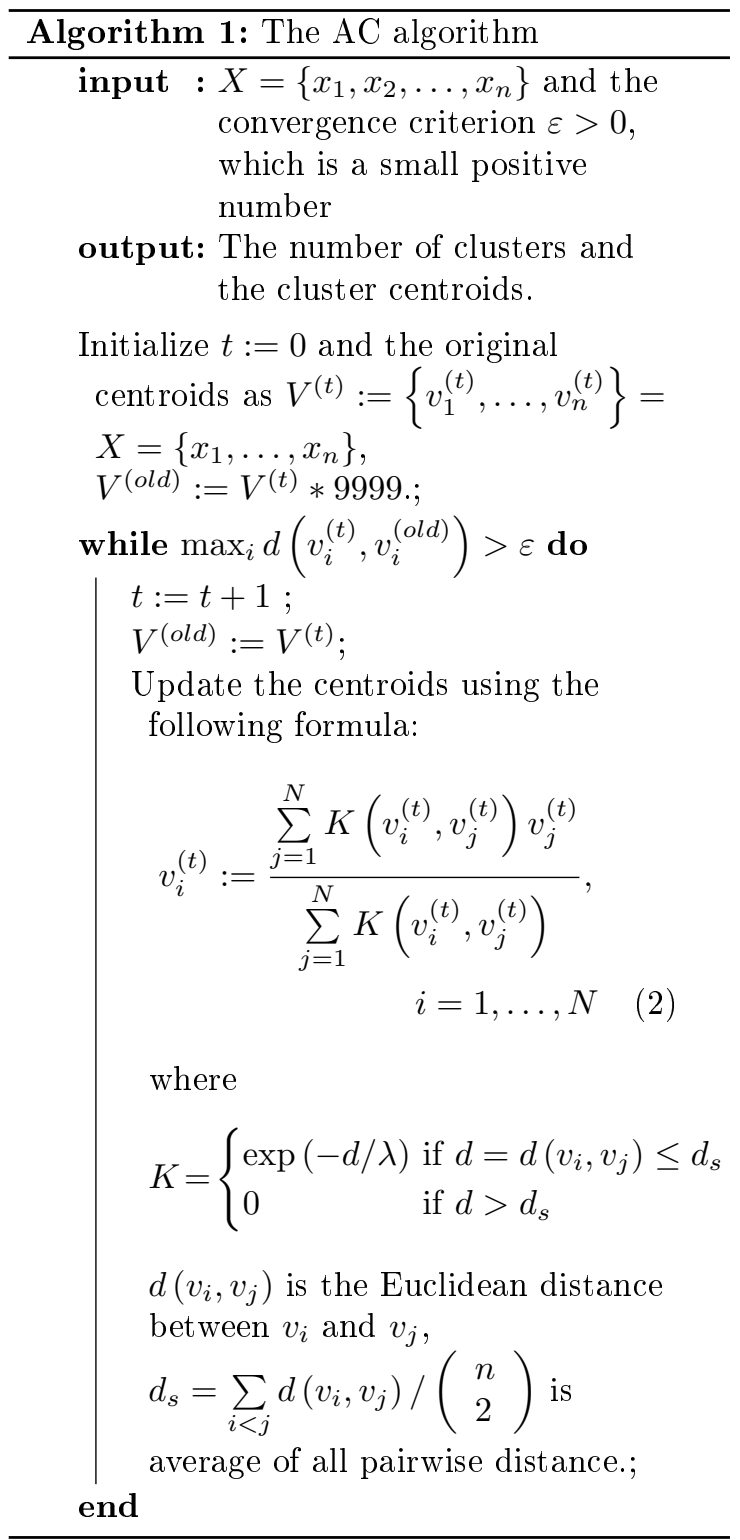

\subsection{The proposed algorithm}

As mentioned above, the $\mathrm{AC}$ is an algorithm with complexity $O\left(n^{2}\right)$. In the case of image contrast enhancement, $n$ represents for the number of pixels which can be up to hundred thousands. Consequently, the $\mathrm{AC}$ algorithm is not computationally efficient and even is unfeasible due to the limitation of personal computer's memory. Therefore, to improve speed and quality of $\mathrm{CE}$, we propose a two-steps Automatic clustering denoted as the 2-AC. Given an image of $n$ pixels, the 2-AC algorithm finds the number of fuzzy sets and locates the critical points based on the intensity distribution of the original image. The 2-AC algorithm is presented as follows.

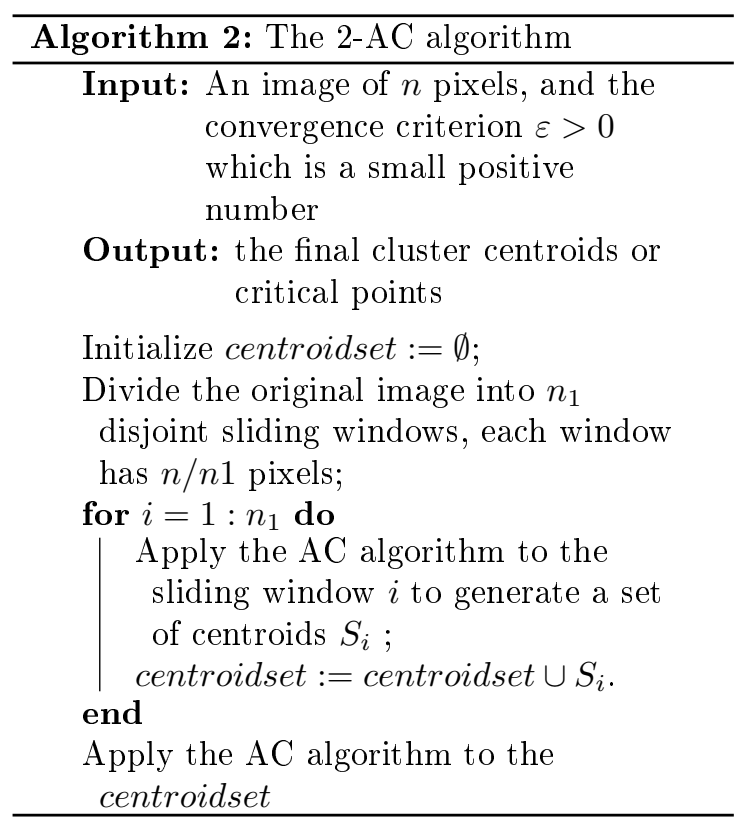

It can be noted from the above pseudo-code that the $\mathrm{AC}$ algorithm is accomplished in two separate steps. In the first step, the $\mathrm{AC}$ algorithm is utilized to find the centroids in $n_{1}$ non-overlapped sliding windows and each sliding window has $n / n_{1}$ pixels. The complexity of this step is $T_{1}(n)=O\left(n_{1}\left(n / n_{1}\right)^{2}\right)=$ $O\left(n^{2} / n_{1}\right)$. Note that, before the loop, we initialize an empty set of centroids. Each time through the loop, we update the centroids set by adding the centroids found in each sliding window. This is a technique often used in "for" loop to find a set of interest points. In the sec- 
ond step, the AC continues to be utilized on the obtained centroids. Let $n_{2}$ be the power of the set of all cluster centroids obtained in the first step, the complexity of the second steps is $T_{2}(n)=O\left(n_{2}^{2}\right)$. It can be implied that the possible maximum value of $n_{2}$ is less than $n$ due to the fact that the value of $\lambda$ is $d s / 10$ and not equal to 0 . Hence, in the worst scenario assumption, the whole complexity of the $2-\mathrm{AC}$ is $T(n)=T_{1}(n)+T_{2}(n)=O\left(\max \left(n^{2} / n_{1}, n_{2}^{2}\right)\right)<$ $O\left(n^{2}\right)$, that is, the 2-AC complexity is less than the original AC. In the best scenario assumption, $n_{2}=n_{1}$, the complexity of the $2-\mathrm{AC}$ is $T(n)=T_{1}(n)+T_{2}(n)=O\left(\max \left(n^{2} / n_{1}, n_{1}^{2}\right)\right)$ and it depends on the number as well as the size of the sliding windows.

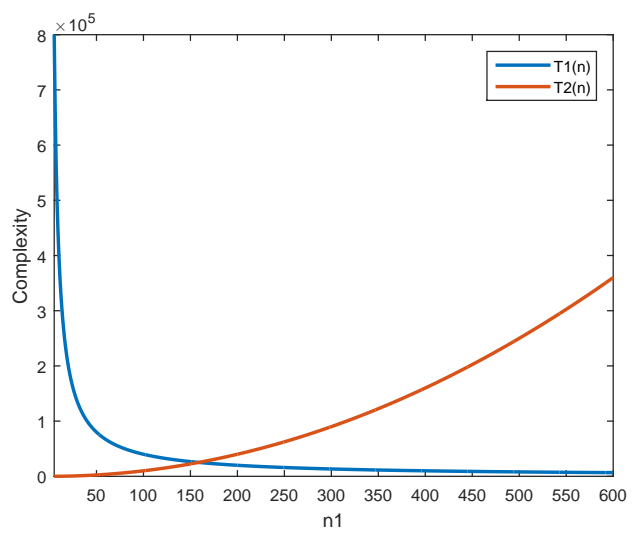

Fig. 2: Illustrating for the Automatic clustering output

In order to better understand that how the complexity depends on the number of sliding windows, Figure 2 illustrates the complexity of the $2-\mathrm{AC}$ in a specific case. It can be implied from Figure 2 that some of the typical choices of the sliding window's resolution like $3 \times 3,5 \times 5$ will lead to a large value of $n_{1}$ and a high complexity $T_{2}(n)$ in the second step as well as a high complexity $T(n)$ in the whole 2-AC algorithm. Also, it can be implied that we can find optimal value of $n_{1}$ that can minimize the value of $T(n)$ by solving the equation $T_{1}(n)=T_{2}(n)$. In this case, the optimal choice of $n_{1}$ is $n^{2 / 3}$, that is, the number of sliding window is $n^{2 / 3}$, the number of pixels in each sliding window is $n^{1 / 3}$ and the whole complexity of the $2-\mathrm{AC}$ is now $O\left(n^{4 / 3}\right)$, which can reduce the computational cost, significantly. In practice, the value
Tab. 1: Some of common standard digital image sizes and the approximate optimal window sizes

\begin{tabular}{rr} 
Photo image size & Sliding window size \\
\hline $1024 \times 768$ & $10 \times 10$ \\
$800 \times 600$ & $9 \times 9$ \\
$512 \times 512$ & $8 \times 8$ \\
$460 \times 308$ & $7 \times 7$ \\
$320 \times 240$ & $6 \times 6$ \\
$256 \times 240$ & $6 \times 6$ \\
$240 \times 160$ & $6 \times 6$ \\
$220 \times 148$ & $6 \times 6$
\end{tabular}

of $n^{1 / 3}$, the optimal number of pixels in each sliding window, is usually not an integer, and therefore this choice is unfeasible. Consequently, for the convenience of computation, the window size of $N \times N$ should be chosen by minimizing the value of $\left|n^{1 / 3}-N^{2}\right|$, where $N$ is a positive integer number. For example, a window size of $6 \times 6$ should be configured for an image of size $220 \times 148$ because $n=220 * 148=32560$ and $\left|n^{1 / 3}-N^{2}\right|=\left|32560^{1 / 3}-6^{2}\right| \simeq 4.07$, which is the minimum of $\left|n^{1 / 3}-N^{2}\right|$ (the corresponding values of $\left|n^{1 / 3}-N^{2}\right|$ for $N=5$ and $N=7$ are 6.93 and 17.07, respectively). Some of the common standard digital image sizes and the approximate optimal window sizes are given in Table 1.

Using the 2-AC algorithm, based on the final centroids, we can determine the number of fuzzy sets $k$ and the critical points in $k$ membership functions. The whole procedure for the 2-ACCE is presented in the following pseudo-code.

\begin{tabular}{l}
\hline Algorithm 3: The 2-ACCE algorithm \\
\hline Input: an image of $n$ pixels and the \\
convergence criterion $\varepsilon>0$, \\
which is a small positive \\
number \\
Output: the enhanced image \\
Utilize the 2-AC algorithm in the \\
whole image for determining the \\
number of fuzzy sets and locating the \\
critical points; \\
Perform the fuzzification phase; \\
Perform the modification phase; \\
Perform the defuzzification phase;
\end{tabular}




\section{Experimental results}

In this section, this article presents two experiments to illustrate and test the performance of the 2-ACCE algorithm. Specifically, Experiment 1 illustrates in detail the 2-ACCE algorithm when performing for the well-known image, the "Lena"; Experiment 2 summarizes the performance of the new method when applied to other natural images in Contrast Enhancement Evaluation Database (CEED2016) [29, 32, 33]. In each experiment, the proposed method is compared with other conventional methods of $[1,17,20]$. This paper uses both global measure as the Root mean squared (RMS) contrast and local measure as the measure of enhancement (EME) to assess the effectiveness of the comparative methods, where higher values of the RMS and EME indicate that better quality the image has. The results are presented as follows.

\subsection{Experiment 1}

In this experiment, the 2-ACCE is applied to the well-known image "Lena" of size $256 \times 240$ (see Figure 3a). Based on Table 1, the image is divided into non-overlapping windows of size $6 \times 6$. Using the $2-\mathrm{AC}$ algorithm, we obtain three final centroids: $v_{1}=0.381, v_{2}=0.467$, $v_{3}=0.553$. Therefore, in the Fuzzification step, we can establish three membership functions as Figure $3 \mathrm{c}$ and transform the image data from gray level domain to the fuzzy membership domain using membership functions. According to [1], in the Modification step, the critical points are modified as $V_{1} \rightarrow V_{1}^{\prime}=0, V_{2} \rightarrow V_{2}^{\prime}=0.5$, $V_{3} \rightarrow V_{3}^{\prime}=1$. In defuzzification step, using Formula (1), we transform the the membership values back into the gray level intensities to achieve the enhanced image as Figure 3b.

Table 2 compares the enhanced images processed by the 2-ACCE and other methods. It can be seen that the enhanced image by [17] is better than that by [1], which is overall not clear. The Yun's method [20] produces largest RMS and EME as compared to the others whereas the 2-ACCE ranks second. However, the enhanced image by the Yun's method is excessively enhanced; as a result, it omits color, and the de-

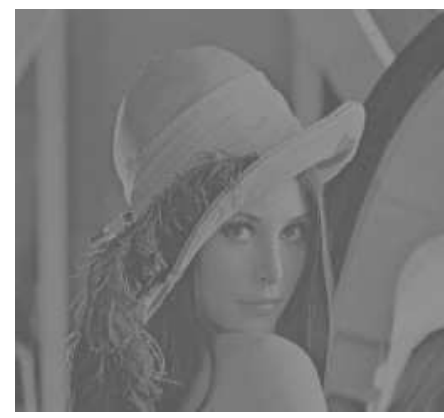

(a) The original image of "Lena"

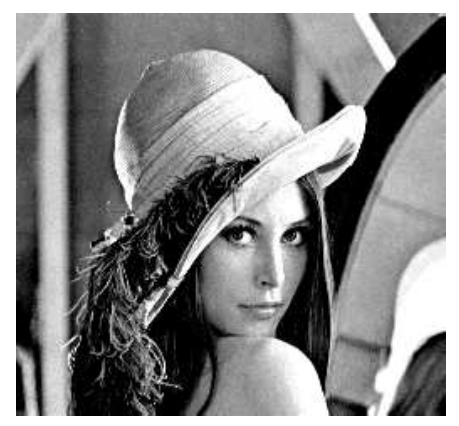

(b) The enhanced image

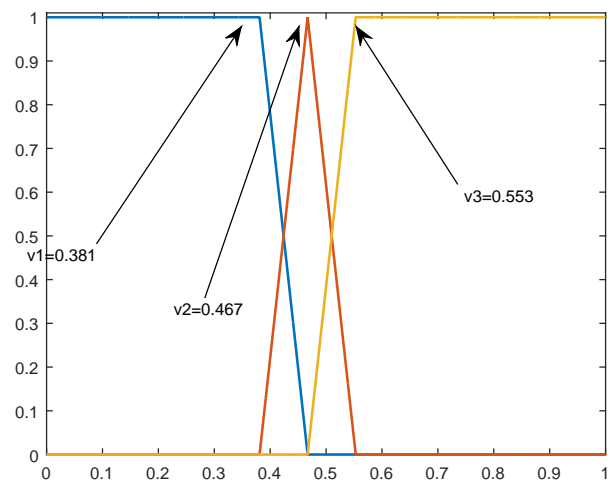

(c) The fuzzy sets and critical points

Fig. 3: Experiment 1 
tails are weakened. On the other hand, the 2ACCE can effectively enhance the contrast of the image with the large RMS and EME, at the same time, it can retain the color information of the original image and improve the visual effect. Compared with the other enhanced images in Table 2, the enhanced image by the 2-ACCE is more brightened and natural looking. Based on the experimental analysis, the 2-ACCE seems to be feasible at first and can be further applied to different natural images in the next experiment.

\subsection{Experiment 2}

In this experiment, the 2-ACCE continues to be tested with other natural images under several conditions of light and background intensity. Specifically, the 2ACCE and other methods are utilized to enhance the quality of images which are made publicly available to the research community at http://wwwl2ti.univ-paris13. fr/site/index.php/en/CEED2016. Figure 4 shows the four original and enhanced images by the proposed method. According to human eyes perception, it can be seen that the 2ACCE algorithm can advance the visibility of all the original images. Furthermore, the $2-\mathrm{ACCE}$ ensures the detail preserving capability; for instance, small details, like the white spots on the dogs in the Image 2, are preserved and look very clear. Table 3 shows the comparisons of the RMS and EME between the 2-ACCE and other conventional methods for the four images. It can be seen that the proposed method provides the highest RMS and EME for all enhanced images in the experiment. That is to say, the 2ACCE can enhance both the global and local contrast. Based on the qualitative and quantitative analyses, it can be implied that the 2-AC has been successfully applied to the $\mathrm{CE}$ and the 2-ACCE method not only has outperformed existing methods in this experiment but also has provided good visual representation.

\section{Conclusion}

This paper proposes a fuzzy rule-based contrast enhancement method using two-steps $\mathrm{Au}-$ tomatic Clustering algorithm. In the first step, the new method performs the Automatic clustering to obtain the centroids in non-overlapped sliding windows and combines them together. In the second step, the Automatic clustering is again applied to the combined centroids set to get the final centroids. Based on the final centroids, the number of fuzzy sets and the critical points of membership functions can be determined and then applied to CE to obtain the enhanced image. The experimental results demonstrate that the new method can effectively enhance the contrast of the images and, at the same time, gives better visual quality images. For future work, it is necessary to apply the proposed method to color image contrast enhancement.

\section{References}

[1] Kannan, P., Deepa, S., \& Ramakrishnan, R. (2012). Contrast enhancement of sports images using two comparative approaches. American Journal of Intelligent Systems, 2(6), 141-147.

[2] Rafael Gonzalez, C., \& Woods, R. (2002). Digital image processing. Michigan, 2nd ed.

[3] Abdullah-Al-Wadud, M., Kabir, M. H., Dewan, M. A. A., \& Chae, O. (2007). A dynamic histogram equalization for image contrast enhancement. IEEE Transactions on Consumer Electronics, 53(2), 593-600.

[4] Babu, P., Rajamani, V., \& Balasubramanian, K. (2015). Multipeak mean based optimized histogram modification framework using swarm intelligence for image contrast enhancement. Mathematical Problems in Engineering, 2015.

[5] Bansal, A., Bajpai, R., \& Saini, J. P. (2007). Simulation of image enhancement techniques using Matlab. In: Modelling \& Simulation, 2007. AMS'07. First Asia International Conference on. IEEE, 296-301. 
Tab. 2: The results of contrast enhancement method

\section{The method of [17] The method of [1] The method of [20] The 2-ACCE}
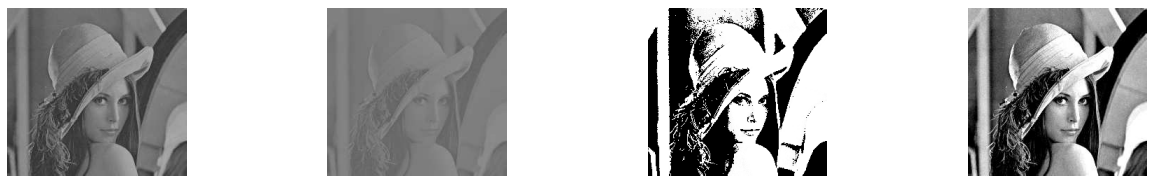

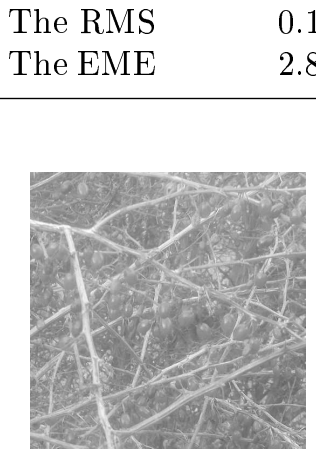

(a) Original Image 1

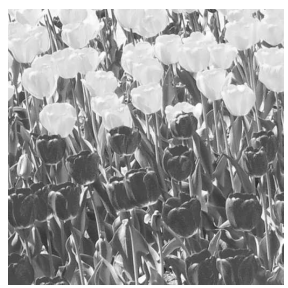

(e) Original Image 3

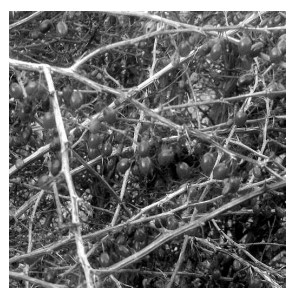

(b) Enhenced Image 1

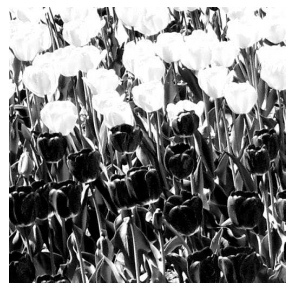

(f) Enhenced Image 3
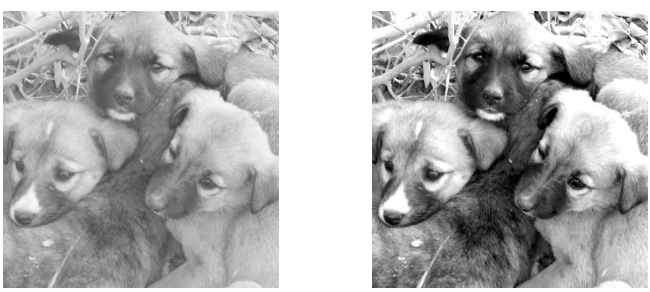

(c) Original Image 2

(d) Enhenced Image 2

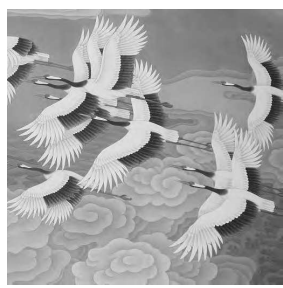

(g) Original Image 5
0.3111

8.7466

Fig. 4: Experiment 2

[6] Chen, S.-D., \& Ramli, A. R. (2003). Minimum mean brightness error bi-histogram equalization in contrast enhancement. IEEE transactions on Consumer Electronics, 49(4), 1310-1319.

[7] Ibrahim, H., \& Hoo, S. C. (2014). Local contrast enhancement utilizing bidirectional switching equalization of separated and clipped subhistograms. Mathematical Problems in Engineering, 2014.

[8] Ibrahim, H., \& Kong, N. S. P. (2007). Brightness preserving dynamic histogram equalization for image contrast enhance- ment. IEEE Transactions on Consumer Electronics, 53(4), 1752-1758.

[9] Kaur, S., \& Kaur, P. (2015). Review and Analysis of Various Image Enhancement Techniques. International Journal of Computer Applications Technology and Research, 4(5), 414-418.

[10] Zadeh, L. A. (1965). Fuzzy sets. Information and control, 8(3), 338-353.

[11] Atanassov, K. T. (1999). Intuitionistic Fuzzy Sets: Theory and Applications. Physica-Verlag HD, Heidelberg, 1-137. 
Tab. 3: Results of comparative methods

\section{RMS}

\begin{tabular}{|c|c|c|c|c|}
\hline & The method of [17] & The method of [1] & The method of [20] & The 2-ACCE \\
\hline Image 1 & 0.1642 & 0.1068 & 0.0922 & 0.2195 \\
\hline Image 2 & 0.2624 & 0.1643 & 0.0698 & 0.2739 \\
\hline Image 3 & 0.3644 & 0.2360 & 0.1528 & 0.3846 \\
\hline \multirow[t]{3}{*}{ Image 4} & 0.2749 & 0.1721 & 0.1022 & 0.2848 \\
\hline & \multicolumn{4}{|c|}{ EME } \\
\hline & The method of [17] & The method of [1] & The method of [20] & The 2-ACCE \\
\hline Image 1 & 4.3962 & 2.9018 & 2.6002 & 10.9786 \\
\hline Image 2 & 3.2033 & 1.7962 & 0.8009 & 4.2659 \\
\hline Image 3 & 9.3860 & 4.2624 & 3.3103 & 10.9175 \\
\hline Image 4 & 4.4318 & 2.9600 & 1.2772 & 5.0381 \\
\hline
\end{tabular}

[12] Cuong, B. C., \& Kreinovich, V. (2013). Picture fuzzy sets - A new concept for computational intelligence problems. In: 2013 Third World Congress on Information and Communication Technologies (WICT 2013). 1-6.

[13] Garg, H. (2018). Analysis of an industrial system under uncertain environment by using different types of fuzzy numbers. International Journal of System Assurance Engineering and Management, 9(2), 525-538.

[14] Hoa, N. V., Vu, H., \& Duc, T. M. (2018). Fuzzy fractional differential equations under Caputo-Katugampola fractional derivative approach. Fuzzy Sets and Systems.

[15] Vovan, T. (2018). An improved fuzzy time series forecasting model using variations of data. Fuzzy Optimization and Decision Making.

[16] Vu, H., \& Dong, L. S. (2015). Initial value problem for second-order random fuzzy differential equations. Advances in Difference Equations, 2015(1), 373.

[17] Hassanien, A. E., \& Badr, A. (2003). A comparative study on digital mamography enhancement algorithms based on fuzzy theory. Studies in informatics and control, 12(1), 21-32.
[18] Kansal, N. K. (2010). Fuzzy techniques for image enhancement. Ph.D. thesis.

[19] Preethi, S., \& Rajeswari, K. (2013). Membership Function modification for Image Enhancement using fuzzy logic. International Journal of Emerging Trends \& Technology in Computer Science, 2(2), 114-118.

[20] Yun, H.-j., Wu, Z.-y., Wang, G.-j., Tong, G., \& Yang, H. (2016). A novel enhancement algorithm combined with improved fuzzy set theory for low illumination images. Mathematical Problems in Engineering, 2016.

[21] Tu, T. N., Hoang, H. N., \& Van, T. V. (2015). Using Fuzzy Logic to Enhance the Large Size Remote Sensing Images. International Journal of Information and Electronics Engineering, 5(6), 464.

[22] Hung, W.-L., \& Yang, J.-H. (2015). Automatic clustering algorithm for fuzzy data. Journal of Applied Statistics, 42(7), 15031518.

[23] Chen, J. H., Chang, Y. C., \& Hung, W. L. (2017). A robust automatic clustering algorithm for probability density functions with application to categorizing color images. Communications in Statistics-Simulation and Computation, (just-accepted). 
[24] Chen, J.-H., \& Hung, W.-L. (2015). An automatic clustering algorithm for probability density functions. Journal of Statistical Computation and Simulation, 85(15), 3047-3063.

[25] Hung, W.-L., Chang-Chien, S.-J., \& Yang, M.-S. (2012). Self-updating clustering algorithm for estimating the parameters in mixtures of von Mises distributions. Journal of Applied Statistics, 39(10), 2259-2274.

[26] Nguyentrang, T., \& Vovan, T. (2017). Fuzzy clustering of probability density functions. Journal of Applied Statistics, 44(4), 583-601.

[27] VoVan, T., \& Nguyen Trang, T. (2018). Similar Coefficient of Cluster for Discrete Elements. Sankhya B.

[28] VoVan, T., \& NguyenTrang, T. (2018). Similar coefficient for cluster of probability density functions. Communications in Statistics - Theory and Methods, 47(8), 1792-1811.

[29] Qureshi, M. A., Beghdadi, A., Sdiri, B., Deriche, M., \& Alaya-Cheikh, F. (2016). A comprehensive performance evaluation of objective quality metrics for contrast enhancement techniques. In: 2016 6th European Workshop on Visual Information Processing (EUVIP). 1-5.

[30] Panetta, K., Gao, C., \& Agaian, S. (2013). No reference color image contrast and quality measures. IEEE Transactions on Consumer Electronics, 59(3), 643-651.

[31] Peli, E. (1990). Contrast in complex images. JOSA A, 7(10), 2032-2040.

[32] Beghdadi, A., Qureshi, M. A., \& Deriche, M. (2015). A critical look to some contrast enhancement evaluation measures. In: 2015 Colour and Visual Computing Symposium (CVCS). 1-6.

[33] Qureshi, M. A., Beghdadi, A., \& Deriche, M. (2017). Towards the design of a consistent image contrast enhancement evaluation measure. Signal Processing: Image Communication, 58, 212-227.

\section{About Authors}

Ha CHE-NGOC works as a lecturer at Faculty of Mathematics and Statistics, Ton Duc Thang University. She graduated B.E. and M.E. degrees in Probability Theory and Mathematical Statistics in 2011 and 2014, respectively. Her research interests are Data mining, Classification, Clustering and Image Processing.

Anh Thy PHAM-CHAU reveived the B.Sc degree in Statistics at Ton Duc Thang University, Ho Chi Minh city, Vietnam. Currently, she has been studying her Master full time at Technical University of Ostrava through a cooperated program between Ton Duc Thang University and Technical University of Ostrava. Her recent interests are towards statistics, machine learning and data mining.

Dibya Jyoti BORA received B.Sc. Honors in Mathematics with distinction from Dibrugarh University, Assam. He received Master of Science in Information Technology with University First Rank from Barkatullah University (Formerly Bhopal University), Bhopal. He received Ph.D. in Computer Science from the Department of Computer Science and Applications, Barkatullah University (Formerly Bhopal University), Bhopal, India. Currently, he is working as an Assistant Professor in the School of Computing Sciences, The Assam Kaziranga University, Jorhat, Assam. His research interest is Image Processing and Machine Learning in broad. And, currently, his research mainly focuses on advanced fuzzy logic based Medical image processing.

"This is an Open Access article distributed under the terms of the Creative Commons Attribution License, which permits unrestricted use, distribution, and reproduction in any medium, provided the original work is properly cited (CC BY 4.0)." 